\title{
Lack of Awareness and Self-Neglect in a Patient with Suspicious Tongue Squamous Cell Carcinoma: Case Report
}

\author{
Mohammad Reza \\ Residency Program Department of Oral Medicine \\ Faculty of Dentistry, Universitas Indonesia \\ Jakarta, Indonesia
}

\author{
Harum Sasanti Yudoyono \\ Department of Oral Medicine \\ Faculty of Dentistry, Universitas Indonesia \\ Jakarta, Indonesia
}

\author{
Gus Permana Subita \\ Department of Oral Medicine \\ Faculty of Dentistry, Universitas Indonesia \\ Jakarta, Indonesia
}

\begin{abstract}
Ninety percent of malignant tumors that present in the mouth are squamous cell carcinoma (SCC). It may affect any anatomical site in the mouth, but most commonly found on the lip, ventral or lateral tongue and the floor of the mouth. These lesions are seen as red, white, red-white, exophytic, or ulcerative lesions. The various problems such as patient personality, social environment and symptoms of the disease can be influenced the awareness and delay of the diagnosis thereby affecting the prognosis of SCC. A suspect case of SCC was found in a 71-years old female who came to Faculty Dental Hospital of Universitas Indonesia with a chief complaint of swollen and easily bleeding gingiva for one month on the left mandible. Intraoral tumor lesions exist in the region 35, 36, 37 of the alveolar left mandible with indentation of tooth antagonists on the dorsal surface and also the irreversible white plaque lesions with reddish erosive lesions on the lateral and ventral left tongue with induration extending to the left floor of the mouth. Chronic gingivitis and radix also founded. For the management, patient was given the information about SCC and motivation involving her family also oral hygiene care instructions. Then the patient was referred to an Oncologist and administered of chlorine dioxide mouthwash and multivitamin. Cytological was performed and malignant squamous cells were discovered. After tumor excision, the patient refused chemotherapy, but unfortunately, she died seven months later. As a conclusion, lack of awareness and self-neglect in SCC is reflected by the extent of the tumor and it requires more aggressive treatment which tailored to patient's condition and involves co-operation of other competent colleagues.
\end{abstract}

Keywords-squamous cell carcinoma, awareness, selfneglect, oral

\section{INTRODUCTION}

Cancer is a non-communicable disease that causes the second greatest death after heart disease and one of the six most common cancers in the world is oral carcinoma [1,2]. Oral carcinoma includes several malignant tumors that occur in the oral region, pharyngeal areas and salivary glands. According to the
World Health Association, oral carcinoma comprises several types including epithelial malignant tumors, salivary gland tumors, soft tissue tumors, hematolymphoid tumors, mucosal malignant melanoma and secondary tumors [3].

However, the term oral carcinoma is to be used with squamous cell carcinoma (SCC). It is estimated that over $90 \%$ of all oral neoplasms are SCC, which is representative and most common in all oral neoplasms [4]. As a subclassification of malignant epithelial tumors, KSS comprises several types: verrucous, basaloid, papillary, spindle, acantholytic and adenosquamos [3,5]. SCC is often asymptomatic, so patients are usually unaware until a progressive lesion develops. SCC appears as a red, white, red-white, exophytic, or ulcerative lesion that persistent in the oral cavity. Commonly the location of SCC of the oral cavity is on the lower lip, floor of the mouth and the ventral or lateral border of the tongue [6].

There are 3 factors to consider for initiating initial SCC treatment: factors related to the characteristics of the primary tumor, which is related to the patient and associated with the treatment team. Early detection and early-stage SCC treatment is the most effective way to improve survival and reduce morbidity, damage, length of treatment, and high maintenance costs. However, the prognosis may differ according to the stage of the disease in the diagnosis and the complexity of the interactions of various factors above $[7,8]$.

Most patients are already in an advanced stage when diagnosing SCC. This is due to a delayed diagnosis consisting of a patient delay or a delay by a physiciandefined as the time interval between the first signs or symptoms perception and the initial evaluation by a physician [9]. Some causes of late diagnosis in people with SCC include; fear of diagnosis and treatment or accustomed to a tumor that usually grows slowly. The lack of awareness of the importance of SCC 
examination is also related to old age, low social environment and inadequate hygiene culture [10].

Self-neglect is an unclear syndrome, characterized by a person's inability to meet basic needs thus posing a threat to his personal health and safety. The condition may manifest because the person is not paying attention to the requirements of nutrition, hygiene and his or her own medical needs as well as related to conditions such as dementia, psychotic disorders, substance abuse, brain disorders and life-threatening infections such as AIDS and cancer [11].

This case report aims to report the management and prognosis of SCC that are influenced by the low awareness and self-neglect aspects that occur in patients.

\section{CASE REPORT}

A 71-year old female came to the Faculty Dental Hospital of Universitas Indonesia (RSKGM-UI) with her family with complaints of swollen gums and bleeds easily from 1 month in the left lower jaw making it difficult to eat and chew. At the urging of her family, the patient would be examined further in RSKGM-UI although at that time the gums felt did not hurt. Previously patients had taken antibiotics amoxicillin and pain relievers obtained from general practitioners (GP) one week ago for treatment with the same complaints. During this patient never complained of pain in his mouth. Patients never had a medical history to the dentist. As a widow who has 5 children, the patient lives with one of her sons. In addition, the patient denies experiencing recurrent aphtae complaints, difficulty swallowing and history of fever, fatigue and weight loss drastically.

On extraoral examination, palpation of the left submandibular lymph node is hard and painless. The intraoral examination found a tumor lesion with $3 \times 2 \mathrm{~cm}$ in the left mandibular alveolar ridge, tooth region 35,36 and 37 with dorsum surface in the form of a tooth antagonist and bleed easily, a hard consistency with diffuse lesion boundary with extensive induration at the left floor of the mouth. There was also an irreversible white lesion on the ventral of the left tongue and multiple lesions of red atrophy and erosive areas surrounded by irreversible white plaque, slippery texture, hard consistency along the left lateral tongue. There is a yellowish white tongue coating on the surface of the dorsum of the tongue, enamel caries 17 , 14 and the focus of oral infection include chronic gingivitis and dental root $16,37,41,42$. This patient also has uncontrolled hypertension disease since 2 years ago. Patients deny regularly consume food preserved, smoked, and no family history of the same disease. Based on the clinical features of the lesions, the working diagnosis is suspected squamous cell carcinoma (SCC)

One week later the patient and her family came back to RSKGM-UI. The patient has not yet continued the examination to the Oncology Department because the patient feels not ready for surgery and the family wants to inquire about the current state of the patient.On intraoral examination there was no significant change in the lesion. The patient carries the instructions on the use of mouthwash but the tongue is not brushed. Patient and family again gave motivation and education about the state of the disease. The patient's family finally decided to continue treatment at Dharma Nugraha Hospital Jakarta

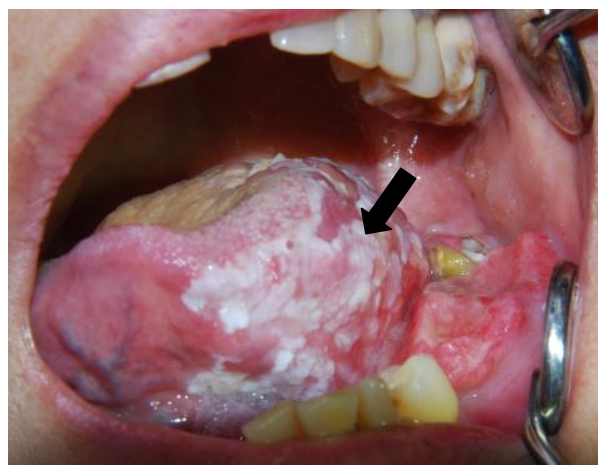

Figure 1. White lesions that can not be lifted with atrophic and erosive areas on the lateral left tongue accompaniedby induration on lateral left tongue.

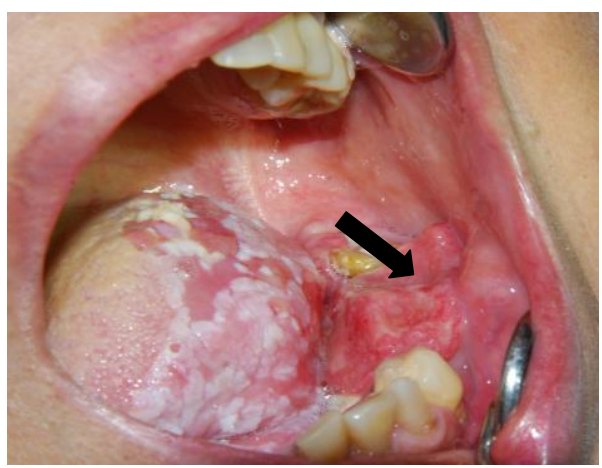

Figure 2. Tumor lesions with dorsum-shaped tooth antagonists in the alveolar ridge region $35,36,37$ with induration extendingto the left floor of the mouth.

Then the patient continued consultation to the Oncologist at the hospital, conducted cytologic examination and found malignant tumor cells of the squamous epithelium. Then the patient performed excision surgery on the tongue and finally planned for chemotherapy. Unfortunately, the patient refused for fear of side effects of chemotherapy and decided not to continue any treatment. The presence of the patient expressed by her family is getting worse. Patients are getting harder to talk, the tongue is getting swollen. Finally, the patient died 7 months later.

\section{CASE MANAGEMENT}

Patient care in the form of motivation and instructions to maintain oral hygiene by brushing teeth twice a day in the morning and night before bed and brush the tongue with soft bristles. On the first visit, the patient and his family were given an explanation of the condition of the illness and the possible risks of the surgical or chemotherapy therapy that would be undertaken. The patient was then referred to Oncology 
and administered 3 times daily chlorine dioxide and multivitamin $1 \mathrm{x}$ daily containing vitamin $\mathrm{C}, \mathrm{E}, \mathrm{B} 1, \mathrm{~B} 2$ B6, B12, and zinc. Patients were also planned to eliminate the oral focus infection by scaling and dental root extraction 16,37,41,42 and improvement of chewing function with a fill cavity 17,14 .

A week later, family and patients came back to consult again about the state of SSC. Patients and families were again given explanations of the suspected SCC disease and were then asked to continue efforts to maintain oral hygiene by continuing the previously given mouthwash and immediately referred to Oncology.

\section{DISCUSSION}

SCC is a common oral cancer that is associated with risk factors that can be prevented. Excessive use of tobacco, betel, and alcohol has been reported as a major etiological factor or social habituation factor. The risk of SCC occurring around $90 \%$ will increase when all these risk factors are used simultaneously. Other risk factors include: extrinsic factors (ultraviolet light, poor oral hygiene, pollution), infection factors (viruses, candida, bacteria), intrinsic factors (genetic, nutritional deficiencies, immunosuppression or immunodeficiency) [12-14].

Patient age and female are globally reported to have a particular prevalence of SCC. This is attributed to differences in certain habits performed by each individual [15]. Globally, in 2012, The estimating incidence of SCC is quite large ie 2.7 cases per 100000 with a value of 3.7 in men and 1.8 in women. The highest proportion was in the Central - South Asia area of $48.7 \%$ and the lowest in North Africa and West Asia at $28.6 \%$ [16].

The patient history of the case report is not informed of any significant habits or risk factors that may contribute to the onset of SCC. But intraoral clinical examination suggests poor oral hygiene factors, chronic gingivitis and dental root that may contribute to the appearance of SCC in the oral cavity. One epidemiological study in India reported that in 480 people with SCC with 774 cases of control, the combined status of oral hygiene of both groups showed $97 \%$ of patients suffered from moderate to severe gingiva inflammation and only 14 were edentulous. Complaints of gum bleeding associated with plaque and calculus show a significant positive correlation with SCC. While in Indonesia, reports indicate poor oral hygiene risk 2.3 times as SCC compared with good oral hygiene. Abandoned oral health due to fear of bleeding gums that do not brush the teeth and the absence of a history of patient visits to the dentist associated with the level of attention of the patient to the condition of the oral cavity and no detection of oral disease from the beginning will have a higher risk of SCC $[17,18]$.

The first come of SCC patient is often followed by a late stage state. This delay in diagnosis is often related to problems that come from the patient or from the examination process treating physician. In this case report, more diagnostic delays are preceded by factors from patients.

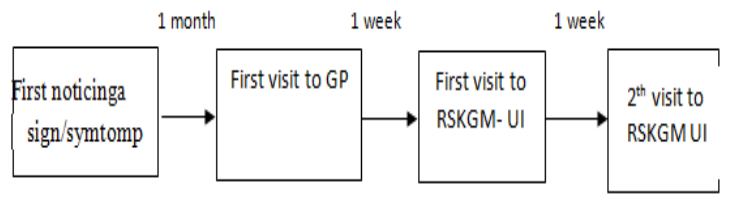

Figure 3. History of patient treatment.

The overall mean survival rate of patients with SCC $\geq$ five years, ie about $50 \%$ of SCC patients with primary stage (I and II ) [19]. While the survival rate of five years at an advanced stage (3 and 4) does not exceed $12 \%$ of cases. Most patients with advanced stage SCC usually die within the first 30 months of SCC. ${ }^{4}$ Poor prognosis is mostly caused by delayed diagnosis, lymph node metastasis and recurrence of SCC [20]. The Jordanian study revealed that low awareness of SCC is due to the low knowledge and level of dental education for SCC signs and risk factors and the importance of early diagnosis and treatment of SCC at $24.1 \%$ in male patients with smoking and alcohol, aged $>40$ years [21]. While other studies in India also showed a low awareness of patients to check into the hospital related to pre-cancerous lesions by $50 \%$ [22].

In this case report, elderly $>70$-year-old patients who live by simply waiting and taking care of households and never going to a dentist become part of a patient's lack of awareness profile which may be followed by a lack of knowledge about SCC disease. It is seen through the information from his family that the patient never complained of pain. However, the history of canker sores that do not heal on the tongue become suspicion of her grandson who had worked in dental institutions to bring the patient to RSKGM-UI. Delayed diagnosis in these patients beforehand is also included with the examination to a GP. This can be understood in terms of the various variables that affect the patient, among others: interpretation of symptoms that are not too concerned (painless, self-reported wound healing), misinterpretation of symptoms, low awareness and decide to try to treat her own illness [23]. Efforts can be made to improve patient knowledge by providing sufficient information through mass media such as; television, newspapers, posters and internet. It also improves the knowledge of dentists against oral cancer through training and can be applied to be delivered to patients who visit in practice $[21,22]$.

Among the different types of cancer, SCC has more psychological and social implications because not only are significant fears and anxieties associated with cancer in general but also affect their functions such as phonetics, taste, and sometimes aesthetics are also affected [11]. In this case, a comprehensive treatment of information and education about the condition of the disease and its treatment plan can improve the spirit of the patient and his family to carry out appropriate care. However, after going through the stage of examination 
and planned for chemotherapy by the Oncology Department, the patient refused for fear of side effects and decided not to continue the treatment.

Hope for the best care by the family cannot be continued due to rejection by the patient. After that, the patient did not make any effort and seemed to wait and did not care. Eventually, the patient's condition worsened as SCC progresses and ends with death. This is known as part of a self neglect attitude. Self-neglect syndrome can be described as one or more of the conditions such as lack of self-care and attention to personal hygiene, apathy and disinterest in personal conditions, withdrawal from the social environment, and strong resistance to aid [24].

Self-neglect is related to medical problems because it affects not only other treatments but in severe cases the potential for death. This strategy of prevention in cancer patients has been studied, although more dominated as an effort to reduce stress and improve quality of life [11]. Approximately $60 \%$ of depression is present in self-neglect syndrome and four times is commonly common in cancer patients from a population, affecting executive function that ends in self-neglect $[11,25,26]$.

Factors related to self-neglect conditions in cancer patients are divided into four main groups namely cancer condition, social environment concern, relationship with religion and condition of death. In the case of cancer, there is a problem of uncertainty in medicine, an approaching death, not knowing the severity of suffering, dependence on others, regrets, unfulfilled desires, inability to accept and helplessness and despair. These aspects trigger an attitude of selfneglect and especially if it continues will fall into the condition of death [11].

Some actions that can be taken to tackle those condition include teaching and negotiating and becoming partner or friends of patients, doing homecare treatments, inviting patients to interact in a supportive social environment and referring patients to psychiatry for evaluation [24].

Lack of awareness and self-neglect in SCC patient is reflected in the spread of the tumor, requiring more aggressive treatment tailored to patient's condition and involving co-operation with other competent partners.

\section{ACKNOWLEDGMENT}

Acknowledgments are addressed to the Minister of Research, Technology and Higher Education of Indonesia, at the expense of this study was derived from DRPM Directorate General of Strengthening Risbang in 2017.

\section{REFERENCES}

[1] World Health Organization, "Chapter 1: Burden: mortality, morbidity and risk factors," Glob. Status Rep. NonCommunicable Dis., vol. 2011, pp. 9-31, 2010.

[2] L. Feller, J. Lemmer, "Oral squamous cell carcinoma: Epidemiology, clinical presentation and treatment," J. Cancer Ther., vol. 3(4), pp. 263-268, 2012.

[3] J.L Tapia, L.J. Goldberg, "The challenges of defining oral cancer: Analysis of an ontological approach," Head Neck
Pathol., vol. 5(4), pp. 376-384, 2011.

[4] K. Markopoulos, "A current aspects on oral squamous cell carcinoma," Open Dent. J., vol. 6(1), pp.126-130, 2012.

[5] T. Maulina, A. Iskandarsyah, A. Hardianto, et al., "The incidence of oral squamous cell carcinoma (OSCC) and its relationship with orofacial pain in oral cancer patients in West Java Province," Indonesia. J. Oral Maxillofac. Surgery Med. Pathol., vol. 29(1), pp. 29-32, 2017.

[6] H. Mortazavi, Y. Safi, M. Baharvand, S. Rahmani, "Diagnostic features of common oral ulcerative lesions: An updated decision tree," Int. J. Dent., 2016.

[7] S. Scott, M. McGurk, E. Grunfeld, "Patient delay for potentially malignant oral symptoms," Eur. J. Oral Sci., vol. 116(2), pp. 141-147, 2008

[8] Shah et al., "Current concepts in management of oral cancerSurgery," Oral Oncol., vol. 45(0), pp. 394-401, 2009.

[9] W. Gao, C.B. Guo, "Factors related to delay in diagnosis of oral squamous cell carcinoma. J. Oral Maxillofac. Surg., vol. 67(5), pp. 1015-1020, 2009.

[10] E. Varga, I. Korom, Z. Raskó, et al., "Neglected basal cell carcinomas in the $21^{\text {st }}$ Century," J. Skin Cancer, pp. 1-4, 2011.

[11] K Nagaraj, A, Khurshid, A.B. Mattoo, "Self-neglect associated with a patient having oral cancer," J. Med. Sci. Clin. Res., vol. 2, pp. 2543-2546, October 2014.

[12] J. Khalili, "Oral cancer: Risk factors, prevention and diagnostic," Exp. Oncol., vol. 30(4), pp. 259-264, 2008.

[13] K. Awan, T. Khang, T. Yee, R. Zain, "Assessing oral cancer knowledge and awareness among Malaysian dental and medical students," J. Cancer Res. Ther., vol. 10(4), pp. 903, 2014.

[14] C. Oji, F. Chukwuneke, "Poor oral hygiene may be the sole cause of oral cancer," J. Maxillofac. Oral Surg., vol. 11(4), pp. 379-383, 2012.

[15] A. Varshitha, "Prevalence of oral cancer in India," Pharm. Sci. Res., vol. 7(10), pp. 845-848, 2015.

[16] K.D. Shield, J. Ferlay, A. Jemal, et al., "The global incidence of lip, oral cavity, and pharyngeal cancers by subsite in 2012," CA Cancer J. Clin., vol. 67(1), pp. 51-64, 2017.

[17] A.M. Sirait, "Faktor risiko tumor/kanker rongga mulut dan tenggorokan di Indonesia," Media Litbangkes, vol. 23(3), pp. 122-129, 2013

[18] P. Sharma, T. Deb, J.G. Ray, et al., "Oral hygiene status of cancer and non cancer patients - a hospital based study, J. Oral Med. Oral Surg. Oral Radiol., vol. 1(2), pp. 57-59, 2015.

[19] D. Marsh, K. Suchak, K.A. Moutasim, et al., "Stromal features are predictive of disease mortality in oral cancer patients," J. Pathol., vol. 223(4), pp. 470-481, 2011.

[20] M.G. Vasconcelos, R.P. Mafra, R.G. Vasconcelos, A. Miryam, C. de Medeiros, "Squamous cell carcinoma of the tongue: clinical and morphological analysis of 57 cases and correlation with prognosis," J. Bras. Patol. e Med., vol. 50, pp. 359-363, October 2014.

[21] Y. Hassona, C. Scully, M. Abu Ghosh, Z. Khoury, et al., "Mouth cancer awareness and beliefs among dental patients," Int. Dent. J., vol. 65(1), pp. 15-21, 2015.

[22] A. Bhoosreddy, S. Bhoosreddy, A. Pandharbale, V. Kunte, et al., "Awareness of patients about existing oral precancerous lesions/conditions in Nashik city of Maharashtra," J. Indian Assoc. Public Heal Dent., vol. 14(2), pp. 207, 2016.

[23] B. Noonan, "Understanding the reasons why patients delay seeking treatment for oral cancer symptoms from a primary health care professional: An integrative literature review," Eur. J. Oncol. Nurs., vol. 18(1), pp. 118-124, 2014

[24] M.P. Pavlou, M.S. Lachs, "Self-neglect in older adults: A primer for clinicians," J. Gen. Intern. Med., vol. 23(11), pp. 1841-1846, 2008.

[25] C.B. Dyer, J.S. Goodwin, S. Pickens-Pace, J. Burnett, P.A Kelly, "Self-neglect among the elderly: A model based on more than 500 patients seen by a geriatric medicine team," Am. J. Public Health, vol. 97(9), pp. 1671-1676, 2007.

[26] M.A. Zimmerman, R.A. Budish, S. Kashyap, S.H. Lindsey, Correlates of depression in self-neglecting older adults: A crosssectional study examining the role of alcohol abuse and pain in increasing vulnerability,” J. Elder. Abus. Negl., vol. 130(12), pp. 1005-1016. 